PROCEEDINGS OF THE

AMERICAN MATHEMATICAL SOCIETY

Volume 140, Number 3, March 2012, Pages 745-747

S 0002-9939(2011)10912-2

Article electronically published on June 8, 2011

\title{
EQUIVARIANT $K$-THEORY AND THE CHERN CHARACTER FOR DISCRETE GROUPS
}

\author{
EFTON PARK
}

(Communicated by Brooke Shipley)

\begin{abstract}
Let $X$ be a compact Hausdorff space, let $\Gamma$ be a discrete group that acts continuously on $X$ from the right, define $\widetilde{X}=\{(x, \gamma) \in X \times \Gamma: x \cdot \gamma=x\}$, and let $\Gamma$ act on $\widetilde{X}$ via the formula $(x, \gamma) \cdot \alpha=\left(x \cdot \alpha, \alpha^{-1} \gamma \alpha\right)$. Results of $\mathrm{P}$. Baum and A. Connes, along with facts about the Chern character, imply that $K_{\Gamma}^{i}(X)$ and $K^{i}(\widetilde{X} / \Gamma)$ are isomorphic up to torsion for $i=0,1$. In this paper, we present an example where the groups $K_{\Gamma}^{i}(X)$ and $K^{i}(\tilde{X} / \Gamma)$ are not isomorphic.
\end{abstract}

Let $\Gamma$ be a finite discrete group acting continuously on a compact Hausdorff space $X$ from the right. Define $\widetilde{X}=\{(x, \gamma) \in X \times \Gamma: x \cdot \gamma=x\}$ and endow $\widetilde{X}$ with the subspace topology that it inherits from $X \times \Gamma$. The group $\Gamma$ acts on $\widetilde{X}$ via the formula $(x, \gamma) \cdot \alpha=\left(x \cdot \alpha, \alpha^{1} \gamma \alpha\right)$, and we can consider the orbit space $\widetilde{X} / \Gamma$. Theorem 1.19 in [1] states that there exist isomorphisms

$$
\begin{aligned}
& K_{\Gamma}^{0}(X) \otimes \mathbb{C} \cong \sum_{j=0}^{\infty} H^{2 j}(\widetilde{X} / \Gamma ; \mathbb{C}), \\
& K_{\Gamma}^{1}(X) \otimes \mathbb{C} \cong \sum_{j=0}^{\infty} H^{2 j+1}(\widetilde{X} / \Gamma ; \mathbb{C}),
\end{aligned}
$$

where $H^{*}(\widetilde{X} / \Gamma ; \mathbb{C})$ denotes the Čech cohomology of $\widetilde{X} / \Gamma$ with complex coefficients. We also have the Chern character isomorphisms

$$
\begin{aligned}
& K^{0}(\tilde{X} / \Gamma) \otimes \mathbb{C} \cong \sum_{j=0}^{\infty} H^{2 j}(\widetilde{X} / \Gamma ; \mathbb{C}), \\
& K^{1}(\tilde{X} / \Gamma) \otimes \mathbb{C} \cong \sum_{j=0}^{\infty} H^{2 j+1}(\tilde{X} / \Gamma ; \mathbb{C}) .
\end{aligned}
$$

Therefore $K_{\Gamma}^{i}(X) \otimes \mathbb{C} \cong K^{i}(\tilde{X} / \Gamma) \otimes \mathbb{C}$ for $i=0$, 1. In fact, a careful reading of [1] shows that we can replace $\mathbb{C}$ by $\mathbb{Q}(\omega)$, where $n$ is the order of $\Gamma$ and where $\omega=\exp (2 \pi i / n)$. In any event, the groups $K_{\Gamma}^{i}(X)$ and $K^{i}(\widetilde{X} / \Gamma)$ are isomorphic up

Received by the editors June 14, 2010 and, in revised form, October 29, 2010; November 16, 2010; and November 23, 2010.

2010 Mathematics Subject Classification. Primary 19L47, 47L65, 19 K99.

Key words and phrases. Equivariant $K$-theory, finite group actions, crossed products.

The author thanks the referee for helpful suggestions.

(C)2011 American Mathematical Society Reverts to public domain 28 years from publication 
to torsion. In this paper, we present an example where these groups do not have isomorphic torsion subgroups.

Our example is Example B in 44. Consider the unit 3 -sphere $S^{3}$ in $\mathbb{R}^{4}$ and define $\alpha: S^{3} \longrightarrow S^{3}$ by $\alpha(x, y, z, t)=(-x,-y,-z, t)$. The map $\alpha$ defines a $\mathbb{Z}_{2}$ action on $S^{3}$. From [2], we know that the $\Gamma$-equivariant $K$-theory groups of a compact Hausdorff space $X$ are isomorphic to the operator algebra $K$-theory groups of $C(X) \rtimes \Gamma$. Combining this fact with the computations in 4 we have $K_{\mathbb{Z}_{2}}^{0}\left(S^{3}\right) \cong \mathbb{Z}^{3}$ and $K_{\mathbb{Z}_{2}}^{1}\left(S^{3}\right) \cong 0$.

Observe that $\widetilde{S^{3}}$ is homeomorphic to the disjoint union of $S^{3}$ and the fixed point set $F$ of the action of $\alpha$, and hence $\widetilde{S^{3}} / \mathbb{Z}_{2}$ is homeomorphic to the disjoint union of $S^{3} / \mathbb{Z}_{2}$ and $F$. In our example, $F$ is the two-point set $\{(0,0,0,1),(0,0,0,-1)\}$, so $K^{0}(F) \cong \mathbb{Z}^{2}$ and $K^{1}(F) \cong 0$.

To compute the $K$-theory of $S^{3} / \mathbb{Z}_{2}$, define closed sets

$$
\begin{aligned}
& A=\left\{(x, y, z, t) \in S^{3}: t \geq 0\right\}, \\
& B=\left\{(x, y, z, t) \in S^{3}: t \leq 0\right\} .
\end{aligned}
$$

Then $\left(A / \mathbb{Z}_{2}\right) \cup\left(B / \mathbb{Z}_{2}\right)=S^{3} / \mathbb{Z}_{2}$ and $\left(A / \mathbb{Z}_{2}\right) \cap\left(B / \mathbb{Z}_{2}\right) \cong S^{2} / \mathbb{Z}_{2} \cong \mathbb{R} P^{2}$. Applying the Mayer-Vietoris sequence for reduced $K$-theory ([3], Exercise 3.2 ), we have the six-term exact sequence

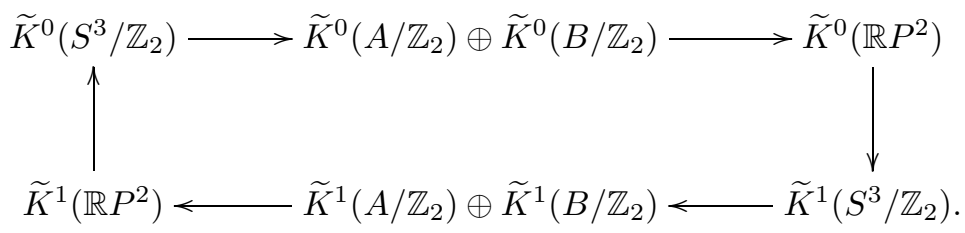

Both $A / \mathbb{Z}_{2}$ and $B / \mathbb{Z}_{2}$ are homeomorphic to the cone over $\mathbb{R} P^{2}$, which has trivial reduced $K$-theory groups, and so the vertical maps in the six-term exact sequence are isomorphisms. Therefore

$$
\begin{gathered}
K^{0}\left(S^{3} / \mathbb{Z}_{2}\right) \cong \widetilde{K}^{0}\left(S^{3} / \mathbb{Z}_{2}\right) \oplus \mathbb{Z} \cong \widetilde{K}^{1}\left(\mathbb{R} P^{2}\right) \oplus \mathbb{Z} \cong \mathbb{Z}, \\
K^{1}\left(S^{3} / \mathbb{Z}_{2}\right) \cong \widetilde{K}^{1}\left(S^{3} / \mathbb{Z}_{2}\right) \cong \widetilde{K}^{0}\left(\mathbb{R} P^{2}\right) \cong \mathbb{Z}_{2},
\end{gathered}
$$

which yield

$$
\begin{gathered}
K^{0}\left(\widetilde{S^{3}} / \mathbb{Z}_{2}\right) \cong K^{0}\left(S^{3} / \mathbb{Z}_{2}\right) \oplus K^{0}(F) \cong \mathbb{Z} \oplus \mathbb{Z} \oplus \mathbb{Z}, \\
K^{1}\left(\widetilde{S^{3}} / \mathbb{Z}_{2}\right) \cong K^{1}\left(S^{3} / \mathbb{Z}_{2}\right) \oplus K^{1}(F) \cong \mathbb{Z}_{2} .
\end{gathered}
$$

Thus $K_{\mathbb{Z}_{2}}^{1}\left(S^{3}\right)$ and $K^{1}\left(\widetilde{S^{3}} / \mathbb{Z}_{2}\right)$ are isomorphic up to torsion, but the groups $K_{\mathbb{Z}_{2}}^{1}\left(S^{3}\right)$ and $K^{1}\left(\widetilde{S^{3}} / \mathbb{Z}_{2}\right)$ are not isomorphic.

\section{REFERENCES}

[1] P. Baum and A. Connes, "Chern character for discrete groups", A Fête of Topology, 163-232, Academic Press, Boston, 1988. MR928402 (90e:58149)

[2] P. Green, "Equivariant K-theory and crossed product $\mathrm{C}^{*}$-algebras", Proc. Symp. Pure Math, no. 38, Part 1 (1982), 337-338. 
[3] E. Park, Complex Topological K-Theory, Cambridge Studies in Advanced Mathematics, Cambridge University Press, Cambridge, 2008. MR2397276 (2009c:19010)

[4] W. Paschke, " $Z_{2}$-equivariant $K$-theory", 362-373, Lecture Notes in Math., no. 1132, Springer, Berlin, 1985. MR799580 (87f:46134)

Department of Mathematics, Box 298900, Texas Christian University, Fort Worth, TEXAs 76129

E-mail address: e.park@tcu.edu 